\title{
Biological significance of miR-126 expression in atrial fibrillation and heart failure
}

\author{
X.J. Wei ${ }^{1}$, M. Han², F.Y. Yang ${ }^{1}$, G.C. Wei ${ }^{1}$, Z.G. Liang ${ }^{1}$, H. Yao ${ }^{1}$, C.W. Ji ${ }^{1}$, R.S. Xie ${ }^{1}$, \\ C.L. Gong ${ }^{1}$ and Y. Tian ${ }^{1}$ \\ ${ }^{1}$ Intensive Care Unit, The People's Hospital of Laiwu City, Laiwu City, Shandong Province, China \\ ${ }^{2}$ Emergency Department, The People's Hospital of Laiwu City, Laiwu City, Shandong Province, China
}

\begin{abstract}
We investigated the biological significance of microRNA-126 (miR-126) expression in patients with atrial fibrillation (AF) and/or heart failure (HF) to examine the possible mechanism of miR-126-dependent AF and development of HF. A total of 103 patients were divided into three groups: AF group (18 men and 17 women, mean age: $65.62 \pm 12.72$ years), HF group (17 men and 15 women, mean age: $63.95 \pm 19.71$ years), and HF-AF group (20 men and 16 women, mean age: $66.56 \pm 14.37$ years). Quantitative real-time PCR was used to measure relative miR-126 expression as calculated by the $2^{-\Delta \Delta C t}$ method. miR-126 was frequently downregulated in the 3 patient groups compared with controls. This reduction was significantly lower in permanent and persistent AF patients than in those with paroxysmal $\mathrm{AF}(\mathrm{P}<0.05, t$-test). Moreover, miR-126 expression was markedly lower in the HF-AF group compared with the AF and HF groups. The 3 patient groups had higher N-terminal prohormone brain natriuretic peptide (NT-proBNP) levels, lower left ventricular ejection fraction (LVEF), larger left atrial diameter, and higher cardiothoracic ratio compared with controls. There were significant differences in NT-proBNP levels and LVEF among the AF, HF, and HF-AF groups. Pearson correlation analysis showed that relative miR-126 expression was positively associated with LVEF, logarithm of NT-proBNP, left atrial diameter, cardiothoracic ratio, and age in HF-AF patients. Multiple linear regression analysis showed that miR-126 expression was positively correlated with LVEF, but negatively correlated with the logarithm of NT-pro BNP and the cardiothoracic ratio (all $\mathrm{P}<0.05$ ). Serum miR-126 levels could serve as a potential candidate biomarker for evaluating the severity of AF and HF. However, to confirm these results, future studies with a larger and diverse patient population are necessary.
\end{abstract}

Key words: MicroRNA-126; Atrial fibrillation; Heart failure; Heart function indices; $\mathrm{N}$-terminal prohormone brain natriuretic peptide (NT-proBNP)

\section{Introduction}

Atrial fibrillation (AF) had a prevalence of more than 2.7 million in the United States in 2010 , with a $25 \%$ increase expected by 2050. However, the age-standardized prevalence of AF is 6.5 per 1000 people in China, which increases with age $(1,2)$. In 2010, AF patients had a high rate of mortality and remaining lifetime risk, and $A F$ was responsible for 107,335 deaths in the United states (1). With regard to heart failure (HF), 5.1 million cases are currently estimated and it is predicted to increase by $46 \%$ from 2012 to 2030 in the US. Similar to AF, HF is associated with a poor 5 -year survival rate, and it accounted for $84 \%$ of overall mortality in 2010 in the United States $(3,4)$.

$\mathrm{AF}$ frequently exacerbates $\mathrm{HF}$, and preexisting $\mathrm{AF}$ is associated with a two-fold higher adjusted heart failure rate in hospitalization for HF. The combination of AF and $\mathrm{HF}$ is an intricate pathophysiological imbalance, which may result in worse morbidity and mortality, poorer quality of life, higher hospitalization rates, and a greater health care burden $(5,6)$. Consequently, increasing investigations have attempted to identify a possible explanation underlying the etiology and development of $\mathrm{AF} / \mathrm{HF}$ because they share many common risk factors, including advancing age, diabetes mellitus, hypertension, myocardial infarction, and valvular heart disease $(7,8)$. In addition, abundant epidemiological data have indicated endogenous causes in the development of $\mathrm{AF} / \mathrm{HF}$, indicating that genetics may contribute to the onset of these diseases $(9,10)$. Intensifying efforts have been made to identify potential biomarkers in early diagnosis of AF/HF. These efforts have led to the recognition of microRNA (miRNA), which participates in disease onset and progression by regulating genes known to be involved in the pathogenesis of $A F / H F(11,12)$.

Correspondence: Guang-Chen Wei: <tianye1013_ty@126.com> or <weichenguang0323@126.com>. 
Numerous lines of evidence have suggested that miRNA plays major roles in regulating the expression of genes that are implicated in the development and pathological progression of cardiovascular diseases by controlling cell differentiation, cell death, cardiac remodeling, fibrosis, vascularization, and cardiomyocyte contraction (13-15). Studies in various models support the possible involvement of differentially expressed miRNAs in the AF/HF process, including miR-1 and miR-133, which increase arrhythmogenesis in HF by dissociating phosphatase activity (16). In addition, the endotheliumspecific miR-126 (also called angiomiR-126) is located within the seventh intron of the EGFL7 gene, which resides on human chromosome 9 . This miRNA plays an important role in angiogenesis and is considered a prognostic biomarker of vascular damage and endothelial dysfunction (17). Importantly, miR-126 is also one of the most abundant miRNAs in human heart, where it may protect against the onset of atherosclerosis by inhibiting vascular cell adhesion molecule-1 protein levels during inflammation (18-20). Moreover, circulating miR-126 is downregulated in acute myocardial infarction and may have the potential for use as a novel biomarker for clinical diagnosis of acute myocardial infarction (21). Furthermore, plasma miR-126 levels are upregulated in HF patients, and miR-126 levels are negatively correlated with B-type natriuretic peptide (BNP) serum levels $(22,23)$. Interestingly, miR-126 is also downregulated in $A F$ patients compared with healthy controls, and may have an effect on diastolic dysfunction in AF, thus suggesting a role for miR-126 in progression of AF (11). Although a worsening of endothelial function, angiogenesis, and diastolic dysfunction can occur in HF and AF, the biological significance of miR-126 downregulation is unknown $(24,25)$. Cellular aging or a perfusion deficiency might decrease renewal of endothelial cells or metabolic activity, which could reduce the release of miR-126 (23). This study aimed to investigate the association of serum miR-126 expression levels with development of AF/HF to determine the biological significance of $m i R-126$ in development of AF/HF.

\section{Material and Methods}

\section{Ethics statement}

This study was approved by the Institutional Review Board of The People's Hospital of Laiwu City. The study protocols complied with the Ethics Guidelines of the 1975 Declaration of Helsinki. Written informed consent was obtained from each patient.

\section{Study population}

A total of 103 patients who were diagnosed with $A F$, $\mathrm{HF}$, and HF plus AF (HF-AF) were admitted to the Department of Cardiology in The People's Hospital of Laiwu City between August 2012 and February 2013. The diagnosis of AF and HF was made based on the 1994 New York Heart Association (NYHA) Functional Classification System by electrocardiogram (26). The AF group $(n=35)$ also included nine patients with paroxysmal AF, 14 with persistent $A F$, and 11 with permanent $A F$ (18 men and 17 women, mean age: $65.62 \pm 12.72$ years). The HF group $(n=32)$ consisted of 18 patients with NYHA III HF and 14 patients with NYHA IV HF (17 men and 15 women, mean age: $63.95 \pm 19.71$ years). The HF-AF group $(n=36)$ was composed of 20 men and 16 women with NYHA III-IV (mean age: $66.56 \pm 14.37$ years). Our study also included 32 individuals with healthy sinus rhythm as a control group (15 men and 17 women, mean age: $58.83 \pm 13.76$ years). Patients were excluded if they had a cerebral vascular accident, acute infection, chronic inflammation, severe arrhythmia, malignant tumor with chemotherapy, liver and kidney function failure, mental disorders, or pregnancy.

\section{Blood sample preparation}

Elbow vein blood samples were acquired early in the morning after fasting. Venous blood samples $(5 \mathrm{~mL})$ were collected in vacuum-dried tubes with RNA-free enzymes. The samples were then separated by centrifugation at $3000 \mathrm{~g}$ for $10 \mathrm{~min}$ at room temperature. Serum was transferred to nuclease-free Eppendorf tubes and stored at $-80^{\circ} \mathrm{C}$ until RNA isolation could be performed. Basic clinical data were collected from all of the participants, including medical history, history of smoking, blood pressure (BP), height, weight, body mass index, routine blood data, liver and kidney function, blood glucose levels, blood lipid levels, blood biochemical index of $\mathrm{N}$-terminal prohormone brain natriuretic peptide (NT-proBNP) levels, electrocardiogram results of the lower left ventricular ejection fraction (LVEF), and X-ray examination results of the left atrial (LA) diameter and the cardiothoracic ratio.

\section{RNA extraction and quantitative real-time PCR}

Briefly, total RNA from frozen serum samples was isolated by using a miRNA easy kit (Aidlab Biotechnologies Co., Ltd., China) and following the manufacturer's instructions. RNA levels were analyzed by using an ultraviolet spectrophotometer (Thermo Co., Ltd., USA) to measure the absorbance at 260 and $280 \mathrm{~nm}$. RNA samples exhibited high purity at $A_{260 / 280}>1.9$. The resulting miRNA was retained for quantitative real-time PCR (qRT-PCR). For qRT-PCR, $5 \mu \mathrm{g}$ of total RNA was reverse-transcribed to synthesize cDNA using the GoScript $^{\mathrm{TM}}$ Reverse Transcription System (Promega Co., Ltd., USA) according to the manufacturer's instructions. Briefly, the reactions involved incubations at $25^{\circ} \mathrm{C}$ for $5 \mathrm{~min}, 42^{\circ} \mathrm{C}$ for $60 \mathrm{~min}$, and $70^{\circ} \mathrm{C}$ for $15 \mathrm{~min}$, and were then maintained at $-20^{\circ} \mathrm{C}$ until further study. The qRT-PCR was performed using GoTaq qPCR Master Mix (Promega Co., Ltd.) with the following conditions: incubation for $2 \mathrm{~min}$ at $95^{\circ} \mathrm{C}$, followed by 40 cycles of annealing at $95^{\circ} \mathrm{C}$ for $15 \mathrm{~s}$, and extension at $60^{\circ} \mathrm{C}$ for $60 \mathrm{~s}$. The miR-126 
PCR primer was designed by Ribobio Co., Ltd. (China). Primers used in this study were as follows: miR-126, 5'-TCGTACCG TGAGTAATA ATGCG-3'; and U6 snRNA, 5'-CTCGCTTCGGCAGCACA-3'.

\section{Data analysis}

Expression of miR-126 was measured with the $2^{-\Delta \Delta C t}$ method and the level of human U6 snRNA was used as an endogenous reference (27). Relative expression of miRNA126 is reported as miRNA-126 relative to an internal control gene, called $\Delta \mathrm{Ct}$. The $\Delta \mathrm{CT}$ and $\Delta \Delta \mathrm{CT}$ values were calculated using the following mathematical formulas: $\Delta \mathrm{Ct}=\mathrm{Ct}_{\text {miRNA-126 }}-\mathrm{Ct}_{\mathrm{U} 6}$ and $\Delta \Delta \mathrm{Ct}=\Delta \mathrm{Ct}_{\text {case }}-\Delta \mathrm{Ct}_{\text {control }}$.

\section{Statistical analysis}

Data are reported as means $\pm \mathrm{SD}$. Statistical analysis was performed using SPSS 18.0 software (SPSS Inc, USA). Comparisons between two groups were performed with the independent-sample $t$-test. Multiple group comparisons were assessed with analysis of variance followed by Bonferroni's or Fisher's LSD post hoc tests. Pearson's correlation analyses between different indices were carried out. $\mathrm{P}<0.05$ was considered to be statistically significant. We also used multiple linear regression analyses to evaluate the independent associations of miR-126 with cardiac function index parameters after adjusting for differences in age, diabetes, drug use, and other confounding factors.

\section{Results}

\section{General clinical data and biochemical indicators}

Differences in the general clinical data among the AF, HF, HF-AF, and control groups are shown in Table 1. There were no significant differences in age, gender, BP, a history of hypertension, or a history of diabetes mellitus among the four groups (all $P>0.05$ ). Moreover, there was no significant difference among the groups when treated with different basic drugs (all $P>0.05$ ). We also examined differences in biochemical indicators among the four groups. There were no significant differences in levels of aspartate aminotransferase, alanine transaminase, creatinine, glucose, white blood cells, total cholesterol, triglycerides, low-density lipoprotein cholesterol, and high-density lipoprotein-cholesterol among the four groups (all $\mathrm{P}>0.05$, Table 2).

\section{Expression of $\mathrm{miR}-126$}

Relative expression of miR-126 was lower in the AF group than in controls $(P<0.01)$. Moreover, we found that miR-126 expression was significantly lower in patients with permanent or persistent AF than in those with paroxysmal AF (all $P<0.05$ ). We also found that miR-126 was frequently downregulated in the HF group compared with the control group $(P<0.01)$. Further analysis was performed to compare relative miR-126 levels between NYHA class III and IV HF patients. Slightly lower miR126 levels were observed in NYHA class IV HF patients than in class III HF patients, but this difference was not significant $(P>0.05)$. Lower miR-126 levels were observed in the HF-AF group than in controls $(P<0.01)$. Additionally, miR-126 expression was markedly lower in the HF-AF group (than in the AF group $(P<0.01)$ and the HF group $(P<0.01$, Table 3$)$.

\section{Comparison of cardiac function indices}

NT-proBNP, LVEF, LA diameter, and the cardiothoracic ratio were assessed among patients in the AF, HF, HF-AF,

Table 1. Comparisons of clinical data among the four groups.

\begin{tabular}{lcccc}
\hline Factors & $\begin{array}{c}\text { AF group } \\
(\mathrm{n}=35)\end{array}$ & $\begin{array}{c}\text { HF group } \\
(\mathrm{n}=32)\end{array}$ & $\begin{array}{c}\text { HF-AF group } \\
(\mathrm{n}=36)\end{array}$ & $\begin{array}{c}\text { Control group } \\
(\mathrm{n}=32)\end{array}$ \\
\hline Age & $65.62 \pm 12.72$ & $63.95 \pm 19.71$ & $66.56 \pm 14.37$ & $58.83 \pm 13.76$ \\
Gender (M/F) & $18 / 17$ & $17 / 15$ & $20 / 16$ & $15 / 17$ \\
Hypertension history (\%) & $14(40.0 \%)$ & $18(56.3 \%)$ & $21(58.3 \%)$ & $15(46.9 \%)$ \\
Diabetes mellitus history (\%) & $8(22.8 \%)$ & $7(21.9 \%)$ & $10(27.7 \%)$ & $7(21.9 \%)$ \\
Smoker $(\%)$ & $9(25.7 \%)$ & $8(25.0 \%)$ & $10(27.7 \%)$ & $6(18.7 \%)$ \\
SBP $(\mathrm{mmHg})$ & $126.6 \pm 12.4$ & $134.2 \pm 18.9$ & $128.5 \pm 14.8$ & $125.5 \pm 15.3$ \\
DBP $(\mathrm{mmHg})$ & $79.2 \pm 8.6$ & $76.8 \pm 10.4$ & $75.4 \pm 9.3$ & $77.3 \pm 10.2$ \\
BMI $\left(\mathrm{kg} / \mathrm{m}^{2}\right)$ & $22.45 \pm 1.89$ & $22.38 \pm 2.01$ & $22.89 \pm 1.65$ & $21.97 \pm 1.87$ \\
Drugs $(\%)$ & & & & \\
$\quad \beta-$ receptor blocker & $21(60.0)$ & $25(78.1)$ & $18(50.0)$ & - \\
Digoxin & $16(45.7)$ & $17(53.1)$ & $22(61.1)$ & - \\
AVEl/ARB & $23(65.7)$ & $15(46.9)$ & $27(75.0)$ & - \\
Loop diuretic & $28(80.0)$ & $25(78.1)$ & $32(88.9)$ & - \\
\hline
\end{tabular}

AF: atrial fibrillation; HF: heart failure; SBP: systolic blood pressure; DBP: diastolic blood pressure; BMI: body mass index; AVEI/ARB: angiotensin enzyme inhibitor/ angiotensin receptor blocker. 
Table 2. Comparisons of biochemical indicators among the groups.

\begin{tabular}{lcccc}
\hline Factors & AF group $(n=35)$ & HF group $(n=32)$ & HF-AF group $(n=36)$ & Control group $(n=32)$ \\
\hline AST $(\mathrm{U} / \mathrm{L})$ & $30.85 \pm 18.50$ & $32.98 \pm 19.62$ & $30.63 \pm 23.16$ & $19.47 \pm 9.74$ \\
ALT $(\mathrm{U} / \mathrm{L})$ & $31.22 \pm 20.42$ & $35.78 \pm 16.45$ & $31.34 \pm 14.74$ & $22.56 \pm 9.64$ \\
Creatinine (uM) & $106.59 \pm 23.12$ & $125.56 \pm 26.37$ & $126.80 \pm 24.37$ & $100.46 \pm 29.81$ \\
Glucose (mM) & $5.72 \pm 1.94$ & $5.89 \pm 1.76$ & $6.14 \pm 2.05$ & $5.52 \pm 1.56$ \\
WBC $\left(\times 10^{9} / \mathrm{L}\right)$ & $8.15 \pm 2.12$ & $7.98 \pm 2.09$ & $8.24 \pm 2.04$ & $7.84 \pm 2.04$ \\
TC $(\mathrm{mM})$ & $4.56 \pm 0.95$ & $4.45 \pm 1.21$ & $4.62 \pm 1.18$ & $4.47 \pm 0.98$ \\
TG $(\mathrm{mM})$ & $1.34 \pm 0.65$ & $1.67 \pm 0.70$ & $1.58 \pm 0.73$ & $1.32 \pm 0.56$ \\
LDL-C (mM) & $2.31 \pm 0.58$ & $2.22 \pm 0.55$ & $2.35 \pm 0.57$ & $2.30 \pm 0.44$ \\
HDL-C (mM) & $1.12 \pm 0.23$ & $1.09 \pm 0.32$ & $1.06 \pm 0.27$ & $1.20 \pm 0.19$ \\
\hline
\end{tabular}

AF: atrial fibrillation; HF: heart failure; AST: alanine aminotransferase; ALT: aspartate aminotransferase; WBC: white blood cell count; TC: total cholesterol; TG: triglycerides; LDL-C: low-density lipoprotein cholesterol; HDL-C: high-density lipoprotein cholesterol. There were no significant differences among groups $(P>0.05$, one-way ANOVA).

and control groups. NT-proBNP levels were significantly higher in the AF group $(P<0.01)$, HF group $(P<0.01)$, and HF-AF group $(\mathrm{P}<0.01)$ compared with controls. Patients in the AF group had lower NT-proBNP levels than those in the HF group $(\mathrm{P}<0.05)$. In addition, NT-proBNP levels in the AF group were significantly lower than those in the HF$A F$ group $(P<0.05)$.

We found that LVEF was higher in controls than in the $A F(P<0.01), H F(P<0.01)$, and HF-AF groups $(P<0.01)$. Additionally, LVEF was higher in the AF group than in the $\mathrm{HF}$ and HF-AF groups (both $\mathrm{P}<0.05$ ). The LA diameter was larger in the $A F(P<0.01), H F(P<0.01)$, and $H F-A F$ groups $(P<0.01)$ than in the control group. A similar difference was found in the cardiothoracic ratio between the AF, HF, HF-AF, and control groups (all $\mathrm{P}<0.01$ ). However, the cardiothoracic ratio showed no significant difference among the AF, HF, and HF-AF groups (all $\mathrm{P}>0.05$, Table 4).

\section{Correlation analysis}

Correlations among NT-proBNP levels, LVEF, LA diameter, and the cardiothoracic ratio with miR-126 expression were performed using Pearson correlation analysis. We

Table 3. Comparisons of serum miR-126 levels among the groups.

\begin{tabular}{lc}
\hline & miR-126 level (fold change) \\
\hline AF group $(n=35)$ & $3.35 \pm 0.67^{\star}$ \\
HF group $(n=32)$ & $1.65 \pm 0.46^{\star \#}$ \\
HF-AF group $(n=36)$ & $0.43 \pm 0.12^{\star \#+}$ \\
Control group $(n=32)$ & $11.44 \pm 2.95$ \\
\hline
\end{tabular}

AF: atrial fibrillation; HF: heart failure; miR: microRNA. ${ }^{*} \mathrm{P}<0.01$, compared to the control group; ${ }^{\#} \mathrm{P}<0.01$, compared to the $\mathrm{AF}$ group; ${ }^{+} \mathrm{P}<0.01$, compared to the HF group (t-test). found that miR-126 expression was negatively associated with LVEF $(r=0.374, P<0.01)$ and the logarithm of NTproBNP $(r=-0.783, P<0.01)$. Similar correlations were also observed in the LA diameter $(r=-0.517, \mathrm{P}<0.01)$ and the cardiothoracic ratio $(r=-0.587, \mathrm{P}<0.01)$. We also found that serum miR-126 levels were negatively correlated with age in HF-AF patients $(r=-0.31, P<0.01$, Table 5$)$.

\section{Multiple linear regression analyses}

After adjustment for confounding factors (gender, age, diabetes mellitus, and smoking), serum miR-126 expression levels were positively correlated with LVEF, and negatively correlated with the logarithm of NT-proBNP and the cardiothoracic ratio (all $\mathrm{P}<0.05$, Table 6 ).

\section{Discussion}

The prevalence of AF is positively correlated with the severity of HF. AF may induce or aggravate HF, and vice versa (28). In recent years, evidence has shown that the mortality of HF-AF patients remains high, and that AF, $\mathrm{HF}$, and HF-AF patients have a poor prognosis (5). Accumulating evidence has suggested that miRNAs are closely related to the progression and prognosis of AF and $\mathrm{HF}$, but the underlying mechanisms are still not completely understood $(29,30)$. Therefore, we aimed to examine serum miR-126 expression levels in patients with $A F$ and/or HF, and to investigate the potential of circulating miR-126 as a serum biomarker in patients with AF and/or HF. We found that serum miR-126 levels in patients in the $A F, H F$, and HF-AF groups were lower than those in the controls. Notably, miR-126 serum levels were lowest in HFAF patients among the four groups. These results indicate that circulating serum miR-126 levels are related to the development, progression, and severity of AF and HF.

MicroRNA-126 is a short non-coding RNA that is derived from the EGFL7 gene (31). Numerous studies 
Table 4. Comparisons of cardiac function indices among the groups.

\begin{tabular}{|c|c|c|c|c|}
\hline Factors & AF group $(n=35)$ & HF group $(n=32)$ & HF-AF group $(n=36)$ & Control group $(n=32)$ \\
\hline $\lg (\mathrm{NT}-\mathrm{p}$ & $3.40 \pm 0.57^{*}$ & $4.07 \pm 0.51^{\star \#}$ & $4.10 \pm 0.37^{\star \#}$ & $2.33 \pm 0.86$ \\
\hline LVEF (\%) & $56.90 \pm 5.67^{*}$ & $48.32 \pm 6.00^{\star \#}$ & $49.92 \pm 6.46^{\star \#}$ & $61.82 \pm 4.23$ \\
\hline LA diameter $(\mathrm{mm})$ & $44.95 \pm 9.65^{*}$ & $45.21 \pm 8.82^{*}$ & $46.69 \pm 8.84^{*}$ & $37.28 \pm 6.00$ \\
\hline Cardiothoracic ratio & $0.62 \pm 0.098^{*}$ & $0.63 \pm 0.078^{*}$ & $0.67 \pm 0.088^{*}$ & $0.50 \pm 0.01$ \\
\hline
\end{tabular}

AF: atrial fibrillation; HF: heart failure; IgNT-proBNP: $\log \mathrm{N}$-terminal prohormone brain natriuretic peptide; LVEF: left ventricular ejection fraction; LA: left atrial. ${ }^{*} \mathrm{P}<0.01$, compared to the control group; ${ }^{\#} \mathrm{P}<0.05$, compared to the AF group (t-test).

have shown that miR-126 levels are highly expressed in vascularized tissues, including the heart, liver, and lungs, and is specifically expressed in endothelial and hematopoietic cells $(32,33)$. Expression levels of miR-126 are mediated by Kruppel-like factor 2a (klf2a), a mechanosensitive zinc finger transcription factor, and may lead to activation of the vascular endothelial growth factor signaling pathway in the endothelium (34). Additionally, miR-126 knockdown may result in impaired endothelial cell migration during the processes of vessel growth, development, and organization, which are closely related to the development of AF and HF (35). Therefore, miR126 could play an important role in the regulation of vascular endothelial growth factor pathway activation. Consequently, abnormal miR-126 expression levels in serum may induce defective angiogenesis and result in an increased risk of $\mathrm{AF}$ and HF. Furthermore, decreased miR-126 expression is associated with the outcome of patients with chronic HF, and it could be helpful in the diagnosis of chronic HF (36).

Another important finding in our study was that patients in the AF, HF, and HF-AF groups had higher NT-proBNP levels, a lower LVEF, a larger LA diameter, and a higher cardiothoracic ratio than the controls. These results suggested that heart function indices, including NT-proBNP levels, LVEF, LA diameter, and the cardiothoracic ratio, were closely associated with development and progression of $\mathrm{AF}$ and $\mathrm{HF}$, which were significantly correlated with the severity of $\mathrm{HF}$ and AF. A previous study reported that NT-proBNP expression could be used as a risk parameter in AF/HF because its expression was positively correlated with an increased risk of HF, stroke, and mortality (37). In addition, NT-proBNP levels are significantly higher in patients with $A F$ than in controls, and thus could serve as a helpful serum biomarker to effectively evaluate heart function in heart diseases (38). To better understand the correlations between serum miR126 levels and heart function indices, we carried out Pearson's correlation analysis to explore the underlying mechanism. This analysis showed that miR-126 serum levels were negatively correlated with LVEF, while they were positively associated with the logarithm of NT-proBNP, LA diameter, and the cardiothoracic ratio.
Previous studies have shown that miR-126 regulates the pathological processes of myocardial hypertrophy, myocardial fibrosis, and changes in myocardial ion channels $(33,39)$, which are significantly associated with heart diseases, including AF and HF. In this respect, decreased serum miR-126 levels may underlie changes in heart function indices, and then induce development of AF and $\mathrm{HF}$

In conclusion, our study shows that serum miR-126 expression levels in patients with AF, HF, and HF-AF are low, especially in those with HF-AF. Moreover, serum miR126 levels are closely correlated with heart function indices, including NT-proBNP levels, LVEF, LA diameter, and the

Table 5. Pearson's correlation analysis between serum microRNA-126 levels and clinical and laboratory characteristics in HFAF patients.

\begin{tabular}{lr}
\hline Factors & \multicolumn{1}{c}{$\mathrm{R}$} \\
\hline Ig(NT-proBNP) & $-0.783^{*}$ \\
LVEF & $0.374^{*}$ \\
LA diameter & $-0.517^{*}$ \\
Cardiothoracic ratio & $-0.587^{*}$ \\
ALT & -0.154 \\
AST & -0.177 \\
BMI & -0.095 \\
Glucose & -0.187 \\
WBC & -0.105 \\
TC & -0.204 \\
TG & -0.196 \\
LDL-C & -0.188 \\
HDL-C & 0.076 \\
CREA & -0.184 \\
Age & $-0.310^{*}$ \\
\hline
\end{tabular}

IgNT-proBNP: log N-terminal prohormone brain natriuretic peptide; LVEF: left ventricular ejection fraction; LA: left atrial; ALT: aspartate aminotransferase; AST: alanine aminotransferase; BMI: body mass index; WBC: white blood cell count; TC: total cholesterol; TG: triglycerides; LDL-C: low-density lipoprotein cholesterol; HDL-C: high-density lipoprotein cholesterol. ${ }^{*} \mathrm{P}<0.01$. 
Table 6. Results of multiple linear regression analyses.

\begin{tabular}{|c|c|c|c|c|}
\hline & \multicolumn{2}{|c|}{ Unstandardized coefficients } & \multirow{2}{*}{$\frac{\text { Standardized coefficients }}{\text { Beta }}$} & \multirow[t]{2}{*}{$t$} \\
\hline & B & Standard error & & \\
\hline Constant & 1.902 & 0.513 & & $3.705^{*}$ \\
\hline LVEF & 0.164 & 0.007 & 0.332 & $23.416^{*}$ \\
\hline $\lg (\mathrm{NT}$-pro BNP) & -1.294 & 0.085 & -0.576 & $-15.234^{*}$ \\
\hline $\mathrm{CR}$ & -5.713 & 0.505 & -0.393 & $-11.311^{*}$ \\
\hline
\end{tabular}

LVEF: left ventricular ejection fraction; IgNT-proBNP: log N-terminal prohormone brain natriuretic peptide; CR: cardiothoracic ratio. ${ }^{*} \mathrm{P}<0.001$.

cardiothoracic ratio. However, the potential limitations of the use of U6 snRNA as an endogenous reference may have affected detection of plasma miRNA expression. Collectively, our data indicated that miR-126 serum levels could

\section{References}

1. Go AS, Mozaffarian D, Roger VL, Benjamin EJ, Berry JD, Blaha MJ, et al. Heart disease and stroke statistics - 2014 update: a report from the American Heart Association. Circulation 2014; 129: e28-e292, doi: 10.1161/01.cir.0000441139.02102.80.

2. Zhou Z, Hu D. An epidemiological study on the prevalence of atrial fibrillation in the Chinese population of mainland China. J Epidemiol 2008; 18: 209-216.

3. Heidenreich PA, Albert NM, Allen LA, Bluemke DA, Butler J, Fonarow GC, et al. Forecasting the impact of heart failure in the United States: a policy statement from the American Heart Association. Circ Heart Fail 2013; 6: 606-619, doi: 10.1161/HHF.0b013e318291329a.

4. Murphy SL, Xu J, Kochanek KD. Deaths: final data for 2010. Natl Vital Stat Rep 2013; 61: 1-117.

5. Aleong RG, Sauer WH, Davis G, Bristow MR. New-onset atrial fibrillation predicts heart failure progression. Am J Med 2014; 127: 963-971.

6. Talajic M, Khairy P, Levesque S, Connolly SJ, Dorian P, Dubuc $M$, et al. Maintenance of sinus rhythm and survival in patients with heart failure and atrial fibrillation. J Am Coll Cardiol 2010; 55: 1796-1802, doi: 10.1016/j.jacc.2010.01.023.

7. De Simone G, Devereux RB, Roman MJ, Chinali M, Barac A, Panza JA, et al. Does cardiovascular phenotype explain the association between diabetes and incident heart failure? The Strong Heart Study. Nutr Metab Cardiovasc Dis 2013; 23: 285-291, doi: 10.1016/j.numecd.2011.06.007.

8. Schnabel RB, Rienstra M, Sullivan LM, Sun JX, Moser CB, Levy $D$, et al. Risk assessment for incident heart failure in individuals with atrial fibrillation. Eur J Heart Fail 2013; 15: 843-849.

9. Ellinor PT, Lunetta KL, Albert CM, Glazer NL, Ritchie MD, Smith AV, et al. Meta-analysis identifies six new susceptibility loci for atrial fibrillation. Nat Genet 2012; 44: 670-675, doi: 10.1038/ng.2261.

10. Skrzynia C, Berg JS, Willis MS, Jensen BC. Genetics and heart failure: a concise guide for the clinician. Curr Cardiol Rev 2015; 11: 10-17, doi: 10.2174/1573403X09666131117170446.

11. Xiao J, Liang D, Zhang $\mathrm{Y}$, Liu $\mathrm{Y}$, Zhang $\mathrm{H}$, Liu $\mathrm{Y}$, et al. MicroRNA expression signature in atrial fibrillation with serve as a potential biomarker for evaluating the severity of $\mathrm{AF}$ and HF. Future studies with a larger number of patients from other populations are necessary to confirm these results. mitral stenosis. Physiol Genomics 2011; 43: 655-664, doi: 10.1152/physiolgenomics.00139.2010.

12. Vogel B, Keller A, Frese KS, Leidinger P, Sedaghat-Hamedani $F$, Kayvanpour E, et al. Multivariate miRNA signatures as biomarkers for non-ischaemic systolic heart failure. Eur Heart $J$ 2013; 34: 2812-2822, doi: 10.1093/eurheartj/eht256.

13. Condorelli G, Latronico MV, Dorn GW. microRNAs in heart disease: putative novel therapeutic targets? Eur Heart $J$ 2010; 31: 649-658, doi: 10.1093/eurheartj/ehp573.

14. Olson EN. MicroRNAs as therapeutic targets and biomarkers of cardiovascular disease. Sci Trans/ Med 2014; 6: 239ps3, doi: 10.1126/scitranslmed.3009008.

15. Fic P, Kowalczuk K, Grabarska A, Stepulak A. [MicroRNA - a new diagnostic tool in coronary artery disease and myocardial infarction]. Postepy Hig Med Dosw 2014; 68: 410-418, doi: 10.5604/17322693.1100348.

16. Belevych AE, Sansom SE, Terentyeva R, Ho HT, Nishijima $\mathrm{Y}$, Martin MM, et al. MicroRNA-1 and -133 increase arrhythmogenesis in heart failure by dissociating phosphatase activity from RyR2 complex. PLoS One 2011; 6: e28324, doi: 10.1371/journal.pone.0028324.

17. Ishizaki T, Tamiya T, Taniguchi K, Morita R, Kato R, Okamoto $\mathrm{F}$, et al. miR126 positively regulates mast cell proliferation and cytokine production through suppressing Spred1. Genes Cells 2011; 16: 803-814, doi: 10.1111/gtc.2011.16.issue-7.

18. Lagos-Quintana M, Rauhut R, Yalcin A, Meyer J, Lendeckel W, Tuschl T. Identification of tissue-specific microRNAs from mouse. Curr Biol 2002; 12: 735-739, doi: 10.1016/S09609822(02)00809-6.

19. Jiang $\mathrm{Y}$, Wang HY, Cao HM, Wang CY, Zhang L, Wang H, et al. Peripheral blood miRNAs as a biomarker for chronic cardiovascular diseases. Sci Rep 2014; 4: 5026.

20. Asgeirsdottir SA, van Solingen C, Kurniati NF, Zwiers PJ, Heeringa $P$, van Meurs $M$, et al. MicroRNA-126 contributes to renal microvascular heterogeneity of VCAM-1 protein expression in acute inflammation. Am J Physiol Renal Physiol 2012; 302: F1630-F1639, doi: 10.1152/ajprenal. 00400.2011. 
21. Long G, Wang F, Duan Q, Chen F, Yang S, Gong W, et al. Human circulating microRNA-1 and microRNA-126 as potential novel indicators for acute myocardial infarction. Int J Biol Sci 2012; 8: 811-818, doi: 10.7150/ijbs.4439.

22. Meng S, Cao JT, Zhang B, Zhou Q, Shen CX, Wang CQ. Downregulation of microRNA-126 in endothelial progenitor cells from diabetes patients, impairs their functional properties, via target gene Spred-1. J Mol Cell Cardiol 2012; 53: 64-72, doi: 10.1016/j.yjmcc.2012.04.003.

23. Fukushima $Y$, Nakanishi $M$, Nonogi H, Goto $Y$, Iwai N. Assessment of plasma miRNAs in congestive heart failure. Circ J 2011; 75: 336-340.

24. Jakob P, Doerries $C$, Briand $S$, Mocharla P, Krankel N, Besler C, et al. Loss of angiomiR-126 and 130a in angiogenic early outgrowth cells from patients with chronic heart failure: role for impaired in vivo neovascularization and cardiac repair capacity. Circulation 2012; 126: 2962-2975, doi: 10.1161/CIRCULATIONAHA.112.093906.

25. Jons C, Joergensen RM, Hassager C, Gang UJ, Dixen U, Johannesen A, et al. Diastolic dysfunction predicts newonset atrial fibrillation and cardiovascular events in patients with acute myocardial infarction and depressed left ventricular systolic function: a CARISMA substudy. Eur $J$ Echocardiogr 2010; 11: 602-607.

26. Raphael C, Briscoe C, Davies J, lan Whinnett Z, Manisty C, Sutton R, et al. Limitations of the New York Heart Association functional classification system and self-reported walking distances in chronic heart failure. Heart 2007; 93: 476-482, doi: 10.1136/hrt.2006.089656.

27. Tomasetti M, Staffolani S, Nocchi L, Neuzil J, Strafella E, Manzella N, et al. Clinical significance of circulating miR-126 quantification in malignant mesothelioma patients. Clin Biochem 2012; 45: 575-581, doi: 10.1016/j.clinbiochem.2012.02.009.

28. Deedwania PC, Lardizabal JA. Atrial fibrillation in heart failure: a comprehensive review. Am J Med 2010; 123: 198-204.

29. Divakaran $\mathrm{V}$, Mann $\mathrm{DL}$. The emerging role of microRNAs in cardiac remodeling and heart failure. Circ Res 2008; 103: 1072-1083, doi: 10.1161/CIRCRESAHA.108.183087.

30. Wakili R, Voigt N, Kaab S, Dobrev D, Nattel S. Recent advances in the molecular pathophysiology of atrial fibrillation. J Clin Invest 2011; 121: 2955-2968, doi: 10.1172/ JCl46315.

31. Musiyenko A, Bitko V, Barik S. Ectopic expression of miR$126^{\star}$, an intronic product of the vascular endothelial EGF-like 7 gene, regulates prostein translation and invasiveness of prostate cancer LNCaP cells. J Mol Med 2008; 86: 313-322.

32. Fish JE, Santoro MM, Morton SU, Yu S, Yeh RF, Wythe JD, et al. miR-126 regulates angiogenic signaling and vascular integrity. Dev Cell 2008; 15: 272-284, doi: 10.1016/ j.devcel.2008.07.008.

33. Harris TA, Yamakuchi M, Ferlito M, Mendell JT, Lowenstein CJ. MicroRNA-126 regulates endothelial expression of vascular cell adhesion molecule 1. Proc Natl Acad Sci U S A 2008; 105: 1516-1521, doi: 10.1073/pnas.0707493105.

34. Nicoli S, Standley C, Walker P, Hurlstone A, Fogarty KE, Lawson ND. MicroRNA-mediated integration of haemodynamics and Vegf signalling during angiogenesis. Nature 2010; 464: 1196-1200, doi: 10.1038/nature08889.

35. Nikolic I, Plate KH, Schmidt MH. EGFL7 meets miRNA-126: an angiogenesis alliance. J Angiogenes Res 2010; 2: 9, doi: 10.1186/2040-2384-2-9.

36. Qiang L, Hong L, Ningfu W, Huaihong C, Jing W. Expression of miR-126 and miR-508-5p in endothelial progenitor cells is associated with the prognosis of chronic heart failure patients. Int J Cardiol 2013; 168: 2082-2088.

37. Hijazi Z, Wallentin L, Siegbahn A, Andersson U, Christersson $\mathrm{C}$, Ezekowitz J, et al. N-terminal pro-B-type natriuretic peptide for risk assessment in patients with atrial fibrillation: insights from the ARISTOTLE Trial (Apixaban for the Prevention of Stroke in Subjects With Atrial Fibrillation). J Am Coll Cardiol 2013; 61: 2274-2284, doi: 10.1016/ j.jacc.2012.11.082.

38. Cai B, Wang L, Liu J, Shi Y, Guo Y. N-terminal pro-Brain natriuretic peptide as a useful biomarker for monitoring prognosis in patients with cardiac valve replacement. J Clin Lab Anal 2011; 25: 149-155, doi: 10.1002/jcla.v25.3.

39. Wei $Y$, Nazari-Jahantigh $M$, Neth $P$, Weber $C$, Schober $A$. MicroRNA-126, -145, and -155: a therapeutic triad in atherosclerosis? Arterioscler Thromb Vasc Biol 2013; 33: 449-454, doi: 10.1161/ATVBAHA.112.300279. 